\title{
Electronic Raman Scattering in Superconducting Cuprates
}

\author{
Andrey V. Chubukov ${ }^{1}$, Dirk K. Morr ${ }^{2}$, and Girsh Blumberg ${ }^{3}$ \\ ${ }^{1}$ Department of Physics, University of Wisconsin, Madison, WI 53706 \\ ${ }^{2}$ NSF Science and Technology Center for Superconductivity and \\ Loomis Laboratory of Physics, University of Illinois at Urbana-Champaign, 1110 W. Green St., Urbana, IL 61801 \\ 3 Bell Laboratories, Lucent Technologies, 700 Mountain Ave., Murray Hill, NJ 07974
}

(received July 22 by M.Cardona)

We show that the novel features observed in Raman experiments on optimally doped and underdoped Bi-2212 compounds in $B_{1 g}$ geometry can be explained by a strong fermionic self-energy due to the interaction with spin fluctuations. We compute the Raman intensity $R(\omega)$ both above and below $T_{c}$, and show that in both cases $R(\omega)$ progressively deviates, with decreasing doping, from that in a Fermi-gas due to increasing contribution from the fermionic self-energy. We also show that the final state interaction increases with decreasing doping and gradually transforms the $2 \Delta$ peak in the superconducting state into a pseudo resonance mode below $2 \Delta$. We argue that these results agree well with the experimental data for Bi-2212.

keywords: A Superconductors, E Inelastic Light Scattering, D Electron Electron Interactions.

The form of the fermionic spectral function in optimally doped and underdoped cuprate superconductors has been the subject of intensive experimental and theoretical studies over the last few years. ARPES and neutron experiments demonstrated that the fermionic spectral function undergoes a substantial evolution with decreasing doping, and for underdoped cuprates is very different from the one in a Fermi-gas (FG) [1]. Raman scattering in $B_{1 g}$ geometry is another spectroscopy to study the electronic properties in this momentum region. For electronic Raman scattering, the intensity $R(\omega)$ is in general given by the imaginary part of the fermionic particlehole bubble at small external momentum $\mathbf{q}$ and finite frequency, weighted with the Raman vertices 2 20 5 . In this paper we focus on the $B_{1 g}$ Raman scattering. In $B_{1 g}$ geometry, the Raman vertex $V_{B_{1 g}} \propto \cos k_{x}-\cos k_{y}$, and the scattering thus mostly probes the vicinity of $(0, \pi)$ 57. Recent Raman experiments on overdoped, optimally doped and underdoped $\mathrm{Bi}-2212$ demonstrated that the $B_{1 g}$ Raman intensity $R(\omega)$ undergoes significant changes with decreasing doping [6 9], and progressively deviates from predictions of a FG theory. Indeed, according to the FG theory, $R(\omega)$ in the normal state is finite only for nonzero external momentum $q$, and vanishes at $\omega>v_{F} q$, where $v_{F}$ is the Fermi velocity [10]. In the superconducting state, $R(\omega)$ is finite due to a particlehole mixing and possesses a peak at $\omega=2 \Delta$ where $\Delta$ is the maximum of the superconducting gap $\Delta_{k}$ [3]. Below $2 \Delta$, the intensity scales as $\omega^{3}$ at small frequencies due to the presence of nodes in the $d$-wave gap [5]. In

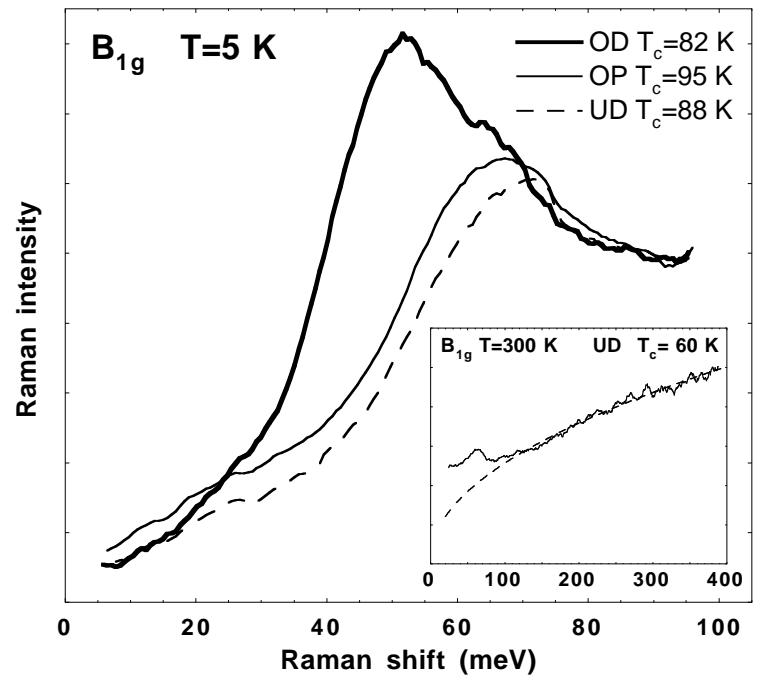

FIG. 1. The $B_{1 g}$ electronic Raman scattering spectra at $T=4 \mathrm{~K}$ for overdoped (OD), optimally doped (OP) and underdoped (UD) $\mathrm{Bi}_{2} \mathrm{Sr}_{2} \mathrm{CaCu}_{2} \mathrm{O}_{8 \pm \delta}$ (Ref. [8]). The inset shows the high energy continuum in the normal state for the underdoped $T_{c}=60 \mathrm{~K}$ sample and its fit to the $\omega^{1 / 2}$ behavior.

Fig. 1 we present the experimental Raman intensity for several $\mathrm{Bi}-2212$ compounds both below and above $T_{c}$ [8]. In the overdoped, $T_{c}=82 \mathrm{~K}$ material, the behavior of $R(\omega)$ is qualitatively consistent with the FG theory, i.e., it is featureless above $T_{c}$ (not shown), while at $T<T_{c}, R(\omega) \propto \omega^{3}$ at small frequencies [11], and exhibits a sharp peak at $2 \Delta \approx 50 \mathrm{meV}$. At and below optimal doping, however, the form of $R(\omega)$ is inconsistent with the FG theory. In the normal state, the intensity increases with frequency as $R(\omega) \sim \sqrt{\omega}$, and saturates at a few hundred meV [7,8,11]. (see the inset in Fig. 1). In the superconducting state, the key experimental observation is that with underdoping, the peak in $R(\omega)$ occurs at progressively smaller frequency than the " $2 \Delta$ " extracted from tunneling experiments [12], and almost saturates at about $75 \mathrm{meV}$ [8]. Simultaneously, the low frequency behavior becomes predominantly linear in $\omega$ with a kink around $40-50 \mathrm{meV}$, while above the peak the intensity develops a dip at about $90 \mathrm{meV}$ and at even larger frequencies recovers the normal state $\sqrt{\omega}$ form.

In this Letter, we make two points. First, we argue that the inconsistency of the experimental results for 

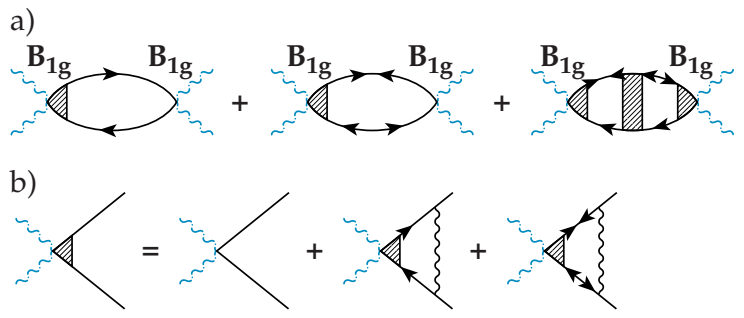

FIG. 2. The full $B_{1 g}$ Raman intensity in a superconductor (a), and the full $B_{1 g}$ Raman vertex (b). The wavy line and dashed rectangle are the fully renormalized effective interaction in $d$-wave and $s$-wave channels, respectively.

Bi-2212 at and below optimal doping with the FG scenario indicates that the fermionic self-energy is large and substantially modifies the form of the fermionic Green's function both in the normal and in the superconducting state. Phenomenologically, this effect was considered in [13. Here we perform a microscopic analysis within a spin-fermion model in which the fermionic self-energy emerges due to an interaction with overdamped spin fluctuations and is the largest for fermions in the vicinity of $(0, \pi)$. Since the Raman intensity in $B_{1 g}$ geometry predominantly probes the region near $(0, \pi)$, it directly reflects the changes in the fermionic spectrum imposed by the large self-energy. Second, we show that a magnetically induced final state interaction replaces the $2 \Delta$ peak in $R(\omega)$ by a pseudo resonance peak at a smaller frequency $\omega_{\text {res }}$. Near optimal doping, this effect is almost unobservable, but it becomes visible in underdoped cuprates and explains the discrepancy between the Raman peak frequency and the one in the density of states.

We now turn to the calculations. The Raman intensity in a superconductor is given by a set of fermionic bubbles made of normal and anomalous Green's functions [14, 15] (Fig.2). We first compute $R(\omega)$ without final state interaction. In this approximation, only the first two diagrams in Fig. 2a contribute (with bare vertices), and we have

$$
\begin{aligned}
& R(\omega) \propto \operatorname{Im} \int d k d \Omega V_{B_{1 g}}^{2}(\mathbf{k}) \\
& \times\left(G_{s c}\left(k, \Omega_{+}\right) G_{s c}\left(k, \Omega_{-}\right)+F\left(k, \Omega_{+}\right) F\left(k, \Omega_{-}\right)\right),
\end{aligned}
$$

where $\Omega_{ \pm}=\Omega \pm \omega / 2$ and

$$
\begin{aligned}
G_{s c}(k, \omega) & =G_{n}^{-1}(-k,-\omega) /\left(G_{n}^{-1}(k, \omega) G_{n}^{-1}(-k,-\omega)+\Delta_{k}^{2}\right) \\
F(k, \omega) & =i \Delta_{k} /\left(G_{n}^{-1}(k, \omega) G_{n}^{-1}(-k,-\omega)+\Delta_{k}^{2}\right) .
\end{aligned}
$$

Here $\Delta_{k}$ is the $d$-wave superconducting gap, and $G_{n}^{-1}(k, \omega)=\omega-\epsilon_{k}+\bar{g}^{2} \Sigma(\mathbf{k}, \omega)$, where $\bar{g}$ is a dimensionless spin-fermion coupling, and $\Sigma(\mathbf{k}, \omega)$ is the $\Delta$-dependent fermionic self-energy. Theoretically, $\bar{g}^{2} \propto \xi^{-1}$ where $\xi$ is the magnetic correlation length [16]. In strongly overdoped cuprates, $\bar{g} \leq 1$, and the system behavior resembles that in a FG. However, at and below optimal doping, $\bar{g} \geq 1$ in which case the self-energy overshadows the bare $\omega$ term in the Green's function, i.e., $G_{n}^{-1}(k, \omega) \approx \bar{g}^{2} \Sigma(k, \omega)-\epsilon_{k}$.

The form of the fermionic self-energy is an input for our Raman calculations. Obviously, the self-energy is the largest near $(0, \pi)$ and symmetry related points where the scattering by nearly antiferromagnetic spin fluctuations is the strongest. The self-energy in this $k$-range also strongly depends on doping as evidenced by ARPES data. Since our goal is to relate the doping dependent changes in $R(\omega)$ with those in $\Sigma(k, \omega)$, we restrict our consideration to the vicinity of $(0, \pi)$ where $\Delta_{k}$ is close to its maximum value $\Delta$. We will argue below that for strong coupling, the $B_{1 g}$ Raman intensity is dominated by $k$ near $(0, \pi)$ down to $\omega \ll \Delta$, and crosses over to $\omega^{3}$ behavior due to the nodes of the d-wave gap only at vanishingly small frequencies.

It has been argued [16,17 that for $\bar{g} \gg 1, \Sigma(k, \omega)$ near $(0, \pi)$ is independent of the quasiparticle energy up to corrections $O\left((\log \bar{g}) / \bar{g}^{2}\right)$, and in a superconductor behaves as $\Sigma(\omega) \propto \omega$ at $\omega \ll 2 \bar{\Delta}$, and as $\Sigma(\omega) \propto$ $e^{i \pi / 4} \sqrt{|\omega|} \operatorname{sgn} \omega$ at $\omega \gg \max \left(2 \bar{\Delta}, \omega_{s f}\right)$, where $\bar{\Delta}=\Delta / \bar{g}$ is a measured gap, and $\omega_{s f} \propto \xi^{-2}$ is a typical relaxation frequency of spin fluctuations. The physical reasoning here is twofold. First, in the normal state, the scattering by nearly-critical overdamped spin fluctuations yields $\Sigma(\omega)=2 \omega /\left(1+\sqrt{1-i|\omega| / \omega_{s f}}\right)$ which displays a crossover from a Fermi-liquid behavior at $\omega<\omega_{s f}$ to a quantum-critical behavior for $\omega>\omega_{s f}$ where $\Sigma(\omega) \propto$ $\sqrt{\omega}$ 16]. Second, in a superconductor, the strength of the scattering by spin fluctuations is reduced below $2 \bar{\Delta}$ due to a feedback effect on the spin damping [17]. The calculation of the full $\Sigma(\omega)$ is rather involved and requires one to solve a set of two coupled complex integral equations for the fermionic self-energy and the spin polarization operator. Below we use the approximate self-consistent solution of this set of equations [17] which correctly reproduces the behavior of $\Sigma(\omega)$ at large and small frequencies. In the latter case it yields $\Sigma(\omega) \approx \omega$, i.e., the peak in the spectral function occurs right at $\omega=\bar{\Delta}$.

We now proceed with $R(\omega)$. We assume that the density of states near $(0, \pi)$ depends only weakly on $\epsilon_{k}$ and replace the $k$-integration in (11) by the integration over $\epsilon_{k}$. We then obtain (neglecting overall prefactor) $R(\omega)=\operatorname{Im} \chi(\omega)$ where

$\chi(\omega)=-\int_{0}^{\infty} d \Omega \frac{\bar{\Delta}^{2}-\Sigma\left(\Omega_{+}\right) \Sigma\left(\Omega_{-}\right)+D\left(\Omega_{+}\right) D\left(\Omega_{-}\right)}{D\left(\Omega_{+}\right) D\left(\Omega_{-}\right)\left(D\left(\Omega_{+}\right)+D\left(\Omega_{-}\right)\right)}+C$,

and $D\left(\Omega_{ \pm}\right)=\sqrt{\Sigma^{2}\left(\Omega_{ \pm}\right)-\bar{\Delta}^{2}}$.

The constant $C>0$ is a real number. Its presence in (3) is related to the fact that Eq. (1) with $k$-integration substituted by the integration over $\epsilon_{k}$ lacks convergence. In this case, one cannot simply interchange frequency and 
energy integrations and has to include a regularization procedure 18. The value of $C$ is irrelevant for the calculations of the Raman intensity without vertex corrections as it does not contribute to $\operatorname{Im} \chi(\omega)$. It however becomes relevant when one includes the effects of the final state interaction which accounts for the renormalization of the Raman vertex (see below). These vertex corrections involve the $d$-wave component of the effective interaction, $\Gamma_{d}$ which decreases at large frequencies and therefore provides the physical regularization of the Raman bubble. In the spin-fluctuation approach, $\Gamma_{d}$ starts decreasing at $\omega \sim \epsilon_{F}^{2} / \bar{g}$ 16]. Another regularization is provided by the fact that the integral over $\epsilon_{k}$ is cut at $\epsilon_{F}$. Obviously, the value of $C$ depends on the ratio $\epsilon_{F} / \bar{g}$. For $\epsilon_{F} \gg \bar{g}$ which is the case at weak coupling, the cutoff in a momentum space dominates. In this situation, an adequate way to evaluate the Raman bubble is to integrate first over frequency. This is how previous calculations of $R(\omega)$ have been performed [3,5]. One then obtains $C=1$. However, in the strong coupling limit $\epsilon_{F} \ll \bar{g}$, which is likely to be satisfied in underdoped cuprates, a more adequate way to evaluate the Raman bubble is to integrate first over $\epsilon_{k}$ as we did. In this case, $C=0$.

We now analyze Eq. (3) first in the normal state and then in the superconducting state. In the normal state, a substitution of the explicit form of $\Sigma(\omega)$ into (3) yields $R(\omega)=R\left(\omega_{s f}\right) \Phi\left(\omega / \omega_{s f}\right)$ where $\Phi(x) \approx 1.07 x$ for $x \ll 1$, and $\Phi(x) \approx 1.73 \sqrt{x}$ for $1 \ll x \ll \bar{g}^{4}$. We see that at $\omega>\omega_{s f}$, relevant to experiments, $R(\omega) \propto \sqrt{\omega}$. We fitted the normal state data by this form and found almost perfect agreement with the experiment (see the inset of Fig. 11). For even larger $x \geq \bar{g}^{4}$, the bare $\omega$ term in the quasiparticle Green's function begins to dominate, and the theoretical $R(\omega)$ saturates, passes through a very broad maximum at $x=2.24 \bar{g}^{4}$ and then slowly decays as $1 / \sqrt{x}$. This also agrees with the data. A similar behavior was found numerically in Ref. [19.

In the superconducting state, the form of $R(\omega)$ clearly depends on the ratio $b=\bar{\Delta} / \omega_{s f}$. Experimentally, $b \leq 1$ in strongly overdoped cuprates, and $b \gg 1$ in strongly underdoped cuprates [1]. For small b, Eq.(3) expectedly reproduces the FG result for the Raman intensity: $R(\omega)=0$ for $\omega<2 \bar{\Delta}$ and $R(\omega) \propto\left(\omega \sqrt{\omega^{2}-4 \bar{\Delta}^{2}}\right)^{-1}$ for $\omega>2 \bar{\Delta}$. The inclusion of the $k$ - dependence of the gap corrects this behavior at small frequencies where it yields a finite $R(\omega) \propto(\omega / \bar{\Delta})^{3}[5]$, and also very near $2 \bar{\Delta}$ where it changes the square root singularity to a logarithmic one. For large $b$, however, the form of the Raman intensity substantially deviates from the FG result. We computed numerically the Raman intensity for different values of $b$, and present the results in Fig. 3. We emphasize four key features in $R(\omega)$ : (i) the $2 \bar{\Delta}$ peak is still present even for large $b$, (ii) with increasing $b$, the peak frequency becomes progressively larger than $2 \bar{\Delta}$, (iii) there is a dip in $R(\omega)$ above the peak, (iv) at small frequencies, $\omega \ll 2 \bar{\Delta}, R(\omega)$ is predominantly linear in $\omega$.

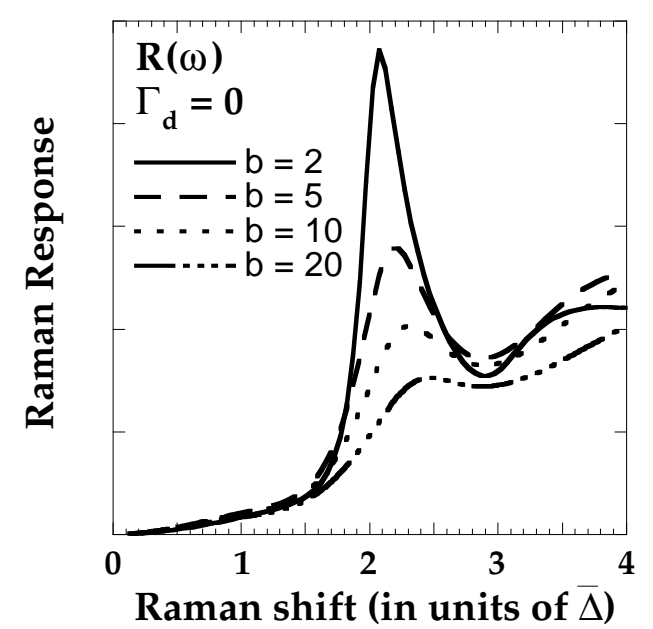

FIG. 3. A calculated Raman intensity, Eqs. (3,99) for different values of $b=\bar{\Delta} / \omega_{s f}$. Larger $b$ correspond to stronger self-energy corrections which destroy fermionic coherence. Observe the broadening of the peak with increasing $b$ and the development of the dip at around $3 \bar{\Delta}$.

These features in the Raman intensity can all be understood analytically by analyzing Eq. (3) in various limits.

The broadening of the peak, the dip and the linear behavior at low frequencies all agree with the features present in the data in Fig. 1. However, one key disagreement with the data remains. Namely, we calculated the density of states $N(\omega)=\int d k \operatorname{Im} G_{s c}(k, \omega)$ along the same lines as the Raman intensity and found that the peak in $N(\omega)$ is located at almost exactly a half of the peak frequency in $R(\omega)$, although both peak frequencies shift to larger values with increasing $b$. The data, we remind, show that with underdoping, the peak in $R(\omega)$ occurs at progressively lower frequencies than twice the peak frequency in $N(\omega)$.

We now argue that the experimentally observed downturn renormalization of the peak in $R(\omega)$ compared to twice that in $N(\omega)$ is due to a fact that the final state interaction between scattered quasiparticles gives rise to a bound state below $2 \bar{\Delta}$.

The final state interaction in both $s$-wave and $d$-wave superconductors have been considered several times in the literature $3,14,15,20$. It gives rise to corrections to the particle-hole vertex (Fig. 20 b), and also introduces an additional scattering process which mixes particle-hole and particle-particle channels [3] 15] (Fig. 2a). A simple experimentation shows that the vertex corrections to the $B_{1 g}$ vertex involve the $d$-wave component of the effective interaction in the zero-sound channel, while the "mixed" diagram (the last one in Fig. 2a) contains the fully renormalized $s$-wave vertex in the particle-particle channel.

Several authors argued 20] that for a $d$-wave superconductor with spin-independent interaction, the effec- 
tive $d$-wave coupling in the zero-sound channel is repulsive, and the bound state does not appear unless the mixed diagram prevails over the conventional, RPA-type vertex renormalization. We, however, demonstrate below that for magnetically-mediated $d$-wave superconductivity there is an additional sign change between vertices in the zero-sound and the Cooper channels, and this eventually gives rise to attractive $d$-wave coupling in the zerosound channel.

Indeed, consider the effective interaction between fermions mediated by the exchange of spin fluctuations with momenta near $Q$. We have $\Gamma=-g^{2} \chi(\mathbf{q}, \Omega) \sigma_{\alpha \beta} \sigma_{\gamma \delta}$ where $\mathbf{q}=\mathbf{k}-\mathbf{k}^{\prime}$ and $\Omega=\omega-\omega^{\prime}$ are transferred momentum and frequency, respectively. To simplify the discussion on the sign of the interaction, assume that $\Gamma$ has a dominant $d$-wave partial amplitude $\Gamma_{d}$, i.e.,

$$
\Gamma\left(k-k^{\prime}, \omega-\omega^{\prime}\right) \approx d_{k} d_{k^{\prime}} \Gamma_{d} \sigma_{\alpha \beta} \sigma_{\gamma \delta}
$$

where $d_{k}$ are $d$-wave eigenfunctions. In general, $\Gamma_{d}$ depends on the transferred frequency. However, one can straightforwardly demonstrate that for a relaxational form of the spin susceptibility, the frequency dependence of $\Gamma_{d}$ becomes relevant at frequencies $\omega \sim \epsilon_{F}^{2} / \bar{g} \gg \bar{\Delta}[16]$. The vertex renormalization on the other hand is mostly determined by much smaller $\omega \sim \bar{\Delta}$. In this situation, $\Gamma_{d}$ can, to a good accuracy, be approximated by a constant.

Let us first verify that there is a superconducting instability in the spin-singlet particle-particle channel. Substituting (4) into the equation for the full particle-particle vertex and making use of the identity

$$
\sigma_{\alpha \beta} \sigma_{\gamma \delta}=T-3 S
$$

where $T, S=\left(\delta_{\alpha \beta} \delta_{\gamma \delta} \pm \delta_{\alpha \delta} \delta_{\gamma \beta}\right) / 2$ are triplet and singlet spin configurations, respectively, we obtain (using for simplicity Fermi gas Green's functions)

$$
\Gamma_{S}^{t o t}=-3 \Gamma_{d} /\left(1+3 \Gamma_{d} L\right)
$$

where $L=\log \left(\omega_{\max } / T\right)>0$. We see that the behavior of the total particle-particle vertex in the spin singlet channel depends on the sign of $\Gamma_{d}$. Several authors have demonstrated that near the antiferromagnetic instability, $\Gamma_{d}<0$ [22]. This obviously implies the superconducting instability. However, if the interaction were spin independent, then the $d$-wave instability would require a positive $\Gamma_{d}$.

Let us turn to the $B_{1 g}$ Raman intensity. The simplest way to check the sign of the vertex correction is to consider a Fermi gas in the normal state, and compute the density-density correlator at zero frequency and at a finite momentum. In the normal state, the mixed diagram does not contribute, and the ladder series of vertex correction diagrams can be easily summed up. We found

$$
V_{B_{1 g}}^{f u l l}(q)=\frac{V_{B_{1 g}}(q)}{1+\Gamma_{d} \chi_{d}(q)}
$$

where $V_{B_{1 g}}$ is the bare $B_{1 g}$ vertex, and

$$
\chi_{d}(q)=\int d \mathbf{k}\left(d_{k}\right)^{2} \frac{\Theta\left(\epsilon_{+}\right)-\Theta\left(\epsilon_{-}\right)}{\epsilon_{+}-\epsilon_{-}}
$$

is the uniform $d$-wave susceptibility $\left(\epsilon_{ \pm}=\epsilon_{k \pm q / 2}\right.$, and $\Theta(x)=1$ when $x>0$ and $\Theta(x)=0$ when $x<0)$. Obviously, $\chi_{d}(q)>0$. We see that for negative $\Gamma_{d}$, the $B_{1 g}$ vertex in the particle-hole channel is enhanced, i.e., the effective interaction in this channel is attractive. We show below that in the superconducting state this attraction gives rise to a pseudo-resonance below $2 \bar{\Delta}$. Previous analytical studies [20] obtained the same expression as in (8), but they considered spin-independent $d$-wave interaction, and therefore set $\Gamma_{d}>0$. In this situation, vertex corrections reduce the $B_{1 g}$ vertex and do not give rise to a resonance behavior.

The conclusion that vertex corrections reduce the $B_{1 g}$ vertex has recently been reached in numerical studies of the Hubbard model 21. The reasons for the discrepancy with our analysis are not clear to us because the effective interaction selected in 21] is apparently also mediated by spin fluctuations.

We now continue our analysis of the superconducting state. The vertex renormalization is given by a set of diagrams in Fig. 2. For a spin-mediated interaction, the $s$-wave coupling is repulsive such that the last diagram in Fig. 2a does not contain a low-energy resonance mode and can be safely neglected. We are then left with the ladder series of vertex correction diagrams. For a frequency independent $\Gamma_{d}$, the series of vertex corrections is then geometrical and yields

$$
R_{f u l l}(\omega)=\frac{\operatorname{Im} \chi(\omega)}{\left(1+\Gamma_{d} \operatorname{Re} \chi(\omega)\right)^{2}+\left(\Gamma_{d} \operatorname{Im} \chi(\omega)\right)^{2}}
$$

where, we remind, $\Gamma_{d}<0$, and $\chi(\omega)$ is given by (3). Now recall that in a superconductor $\operatorname{Im} \chi(\omega)$ is small at $\omega<2 \bar{\Delta}$. Evaluating $\operatorname{Re} \chi(\omega)$ we found that it is positive below $\omega<2 \bar{\Delta}$. Thus for small frequencies, we found $\operatorname{Re} \chi(\omega)=A(\omega / \bar{\Delta})^{2}$ where in a FG, $A=1 / 3$. Substituting this result into (9), we find that $R_{\text {full }}$ possesses a resonance peak below $2 \bar{\Delta}$, at a frequency $\omega=\omega_{\text {res }}$ where $\left|\Gamma_{d}\right| \operatorname{Re} R\left(\omega_{\text {res }}\right)=1$. Actually, the solution for $\omega_{\text {res }}$ exists already at weak coupling simply because in a FG, $\operatorname{Re} \chi(\omega)$ diverges logarithmically as $\omega$ approaches $2 \bar{\Delta}$ from below. As the coupling increases, the peak position progressively deviates downwards from $2 \bar{\Delta}$. A similar reasoning has been previously applied to explain the presence of the resonance peak in the neutron scattering data below $T_{c}$ 23].

Indeed, the bound state which we found is only a pseudo resonance because in a $d$-wave superconductor, $\operatorname{Im} \chi(\omega)$ is finite for all $\omega \neq 0$ because of the nodes of the gap. On the other hand, the very existence of the peak only requires a reduction of $\operatorname{Im} \chi(\omega)$ below $2 \bar{\Delta}$ which is a natural consequence of a reduction of the fermionic 


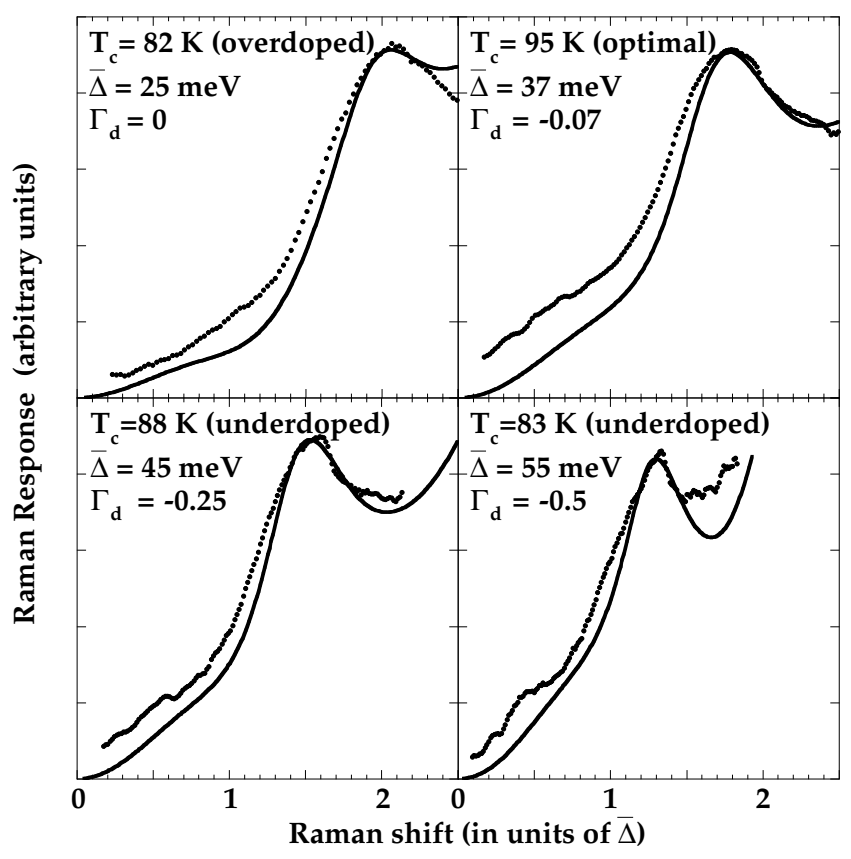

FIG. 4. The fits of the theoretical Raman intensity with final state interaction, $R_{\text {full }}(\omega)$, Eq. 9 to the experimental data for slightly overdoped, optimally doped and underdoped Bi2212 materials. We use the theoretical fact that in the absence of the final state interaction, the peak frequency in the Raman intensity, even at strong coupling, is almost exactly twice the peak frequency in the density of states, and extract an effective $\bar{\Delta}$ from the photoemission and tunneling data [1, 12]. The parameter $\Gamma_{d}$ measures the strength of the vertex corrections which, we argue, give rise to a pseudo-resonance in $R_{\text {full }}(\omega)$. Observe that with underdoping, the peak in $R_{\text {full }}(\omega)$ progressively deviates down from $2 \bar{\Delta}$. The presented fits are for $b=20$. Fits using smaller $b$ reproduce the shape of $R(\omega)$ but yield sharper peaks than in the data.

spectral weight at low frequencies in a superconductor. Moreover, as the spectral weight reduction occurs already in the pseudogap regime [1], our theory predicts that the Raman peak should survive above $T_{c}$ and disappear only at a temperature where the pseudogap behavior becomes invisible.

In Fig. 田we fitted the data from Fig. 1 to Eq. (9) using $\Gamma_{d}$ as an adjustable parameter. We found that in overdoped cuprates, the pseudo resonance frequency is almost indistinguishable from twice the peak frequency extracted from the tunneling data [12]. However, as the system moves towards lower doping, $\left|\Gamma_{d}\right|$ obviously increases, and the peak in $R_{\text {full }}(\omega)$ moves to progressively lower frequencies compared to twice the peak frequency of the tunneling data.

One more point. For $s$-wave case, weak coupling calculations [15] have shown that for an attractive zerosound coupling, $R_{\text {full }}(\omega)$ has two peaks, one at $\omega_{\text {res }}$ and another near $2 \Delta$. We did not find indications for a two peak structure in $R_{\text {full }}(\omega)$. The reason is that in our case, the peak in $R(\omega)$ is already rather broad. Furthermore, we found numerically that the overall shape of $R_{f u l l}(\omega)$ does not change much compared to that in $R(\omega)$, i.e., the dip and the linear behavior at small frequencies are present also in $R_{\text {full }}(\omega)$ (see Fig. 3). ¿From this perspective, the shape of the Raman intensity is mostly determined by the self-energy corrections, while the position of the Raman peak is determined by the resonance in the Raman vertex.

To summarize, we have demonstrated that the experimentally observed doping evolution of the $B_{1 g}$ Raman intensity in cuprates can be explained by an interaction with spin fluctuations. We argued that for the optimally doped and underdoped materials our results capture all salient features of the experimental data in Fig.位: (i) the $\sqrt{\omega}$ behavior of the intensity in the normal state, (ii) a predominantly linear low frequency behavior of $R(\omega)$ in a superconductor, (iii) a reduction of the peak amplitude with decreasing doping and a development of a dip above the peak, and (iv) a progressive downturn deviation of the Raman peak position compared to the distance between the peaks in the tunneling density of states. Finally, the prediction that the Raman peak survives in the pseudogap regime is also consistent with the data [3].

An issue which we didn't address in this paper is the experimentally observed strong discrepancy between the Raman data in $B_{1 g}$ and $A_{1 g}$ geometries [24]. This discrepancy (e.g., different locations of the peak frequencies) is still unexplained [25] and clearly calls for more theoretical work on Raman scattering.

It is our pleasure to thank T. Devereaux, R. Gatt, R. Joynt, M. V. Klein, A. Millis, H. Monien, D. Pines and J. Schmalian for useful conversations. The research was supported by NSF grant DMR-9629839 (A.C.) and by the NSF cooperative agreement DMR91-20000 through STCS (D.M. and G.B.).

[1] M. R. Norman et al., Nature 392, 157 (1998); Z-X. Shen et al., Science 280, 259 (1998); J. Rossat-Mignod et al, Physica C (Amsterdam) 185-189, 86 (1991); H.F. Fong et al, Phys. Rev. B54, 6708 (1996).

[2] A.A. Abrikosov and V.M. Genkin, Sov. Phys. JETP 38, 417 (1974).

[3] M.V. Klein and S.B. Dierker, Phys. Rev. B 29, 4976 (1984).

[4] B.S. Shastry and B.I. Shraiman Phys. Rev. Lett. 65, 1068 (1990); R.R.P. Singh, Cond. Mat. Phys. 15, 241 (1991).

[5] T.P. Devereaux et al., Phys. Rev. Lett. 72, 396 (1994); Phys. Rev. B 54, 12523 (1996); D. Branch and J.P. Carbotte, ibid. 52, 603 (1995); T. Strohm and M. Cardona, ibid. 55, 12725 (1997) and references therein. 
[6] C. Kendziora and A. Rosenberg, Phys. Rev. B 52, R9867 (1995).

[7] R. Hackl et al. J. of Low Temp. Phys. 105, 733 (1996);

R. Nemetschek et al. Phys. Rev. Lett. 78, 4837 (1997).

[8] G. Blumberg et. al., Science 278, 1427 (1997); J. Phys. Chem. Solids 59, 1932 (1998).

[9] J.W. Quilty et al., Phys. Rev. B 57, R11 097 (1998).

[10] P.M. Platzman, Phys. Rev. 139, A379 (1965).

[11] T. Staufer et al., Phys. Rev. Lett. 68, 1069 (1992); T. Staufer, R. Hackl, and P. Müller, Solid State Comm. 79, 409 (1991); D. Reznik et al. Phys. Rev. B 46, 11725 (1992).

[12] Ch. Renner et al, Phys. Rev. Lett. 80, 149 (1998).

[13] C.M. Varma et al., Phys. Rev. Lett. 63, 1996 (1989); G. Kotliar et al. Europhys. Lett. 15, 655 (1991).

[14] J.R. Schrieffer Theory of Superconductivity, Frontiers in Physics, Addison Wesley (1988); A. Bardasis and J.R. Schrieffer, Phys. Rev. 121, 1050 (1961).

[15] H. Monien and F. Zawadowski, Phys. Rev. B 41, 8798 (1990).

[16] A. Chubukov, Europhys. Lett, 44, 655 (1998).

[17] A. V. Chubukov and D. K. Morr, Phys. Rev. Lett. 81, 4716 (1998).

[18] A. Virostek and J. Ruvalds, Phys. Rev. B 45, 347 (1992).

[19] T.P. Devereaux and A.P. Kampf, cond-mat/9711039. See also C. Jiang and J.P. Carbotte, Phys. Rev. B 53, 11868 (1996).

[20] W-C Wu and A. Griffin, Phys. Rev. B 51, 1190 (1995); T.P. Devereaux and D. Einzel, ibid, 51, 16357 (1995).

[21] T. Dahm, D. Manske and L. Tewordt, Phys. Rev. B 59, 14740 (1999) and references therein.

[22] Ph. Monthoux and D. Pines, Phys. Rev. B 47, 6069 (1993); D.J. Scalapino, Phys. Rep. 250, 329 (1995).

[23] I. Mazin and V. Yakovenko, Phys. Rev. Lett. 75, 4134 (1995)); A. Millis and H. Monien, Phys. Rev. B 54, 16172 (1996). A. Abanov and A. Chubukov, Phys. Rev. Lett., August 23 (1999).

[24] T. Strohm and M. Cardona, Phys. Rev. B 55, 12725 (1997) and references therein.

[25] see e.g., T. Strohm et al, Phys. Rev. B 58, 8839 (1998); D. Manske et al, ibid, 58, 8841 (1998); 56, R2940 (1997). 